\title{
Testing for Chlamydia trachomatis: time trends in positivity rates in the canton of Basel-Stadt, Switzerland
}

\author{
C. SCHMUTZ ${ }^{1,2}$, D. BURKI ${ }^{3}$, R. FREI ${ }^{4}$, M. MÄUSEZAHL-FEUZ ${ }^{5}$ \\ AND D. M $\ddot{A} U S E Z A H L^{1,2 *}$ \\ ${ }^{1}$ Swiss Tropical and Public Health Institute, Basel, Switzerland \\ ${ }^{2}$ University of Basel, Basel, Switzerland \\ ${ }^{3}$ Viollier AG, Allschwil, Switzerland \\ ${ }^{4}$ University Hospital Basel, Basel, Switzerland \\ ${ }^{5}$ Swiss Federal Office of Public Health, Bern, Switzerland
}

Received 13 June 2012; Final revision 19 September 2012; Accepted 21 October 2012; first published online 19 November 2012

\section{SUMMARY}

National health statistics report a $2 \cdot 5$-fold increase in laboratory-confirmed Chlamydia trachomatis $(\mathrm{CT})$ cases over the last decade in Switzerland where no CT screening programme exists. We obtained essential denominator information to describe the epidemiology of CT in the canton of Basel-Stadt, an urban canton in north-western Switzerland. Laboratories reporting at least two CT infections from Basel-Stadt residents to the SFOPH in 2010 provided demographic and test-related data. CT positivity rates were calculated for 2002-2010. The influences of test year, age, sex and laboratory on CT positivity were investigated in a multivariable model. Positivity differed between sexes and age groups. In our sample of 32034 records, female and male CT positivity rates were $4 \cdot 7 \%$ and $11 \cdot 1 \%$, respectively. Test year was significantly associated with test outcome in the multivariable analysis but no time trend was observed. CT positivity did not change over the past 9 years in Basel-Stadt. In contrast to other European countries without CT screening, we found no evidence that the observed increase of Chlamydia cases in the national notification system represents an epidemiological trend, but rather results from an increased testing frequency.

Key words: Chlamydia trachomatis, positivity rate, surveillance, Switzerland, time trend.

\section{INTRODUCTION}

In Switzerland and other European countries, an increase in sexually transmitted infections (STIs) has been observed since the end of the 1990s [1]. The most frequent bacterial STI, Chlamydia trachomatis (CT),

\footnotetext{
* Author for correspondence: Dr D. Mäusezahl, Swiss Tropical and Public Health Institute, Socinstrasse 57, PO Box, CH-4002 Basel, Switzerland.

(Email: daniel.maeusezahl@unibas.ch)
}

mainly affects young adults with multiple sexual partners not using condoms [2]. Treatment with antibiotics is effective, but the frequently asymptomatic nature of infections makes it difficult to detect them $[3,4]$.

The WHO estimated that 92 million people worldwide were newly infected with CT in 1999, of which five million were in Western Europe [5]. The epidemiology of CT infections in Switzerland cannot be fully understood because existing studies on disease

The online version of this article is published within an Open Access environment subject to the conditions of the Creative Commons Attribution-NonCommercial-ShareAlike licence $<$ http://creativecommons.org/licenses/by-nc-sa/2.5/ $>$. The written permission of

Cambridge University Press must be obtained for commercial re-use. 
prevalence focus on selected risk groups such as prisoners [6], undocumented immigrants [7] or men at army recruitment [8].

Since 1988, laboratories are obliged by the Swiss Epidemics Act to report positive Chlamydia test results to the Swiss Federal Office of Public Health [SFOPH/Bundesamt für Gesundheit (BAG)] [9]. All CT infections diagnosed by culture, genome (DNA/ RNA) or antigen detection from genital sites must be reported. Currently, predominantly nucleic acid amplification tests (NAATs) are used for the diagnosis of CT infections [10]. Figures from the national notification system for infectious diseases (NNSID) indicate a threefold increase of CT cases over the past 12 years [11]. Negative test results are not reported to SFOPH and consequently no denominator data is available for appropriate epidemiological trend analysis. An increase in disease notifications is often considered to reflect an increase in disease frequency. We questioned this interpretation and hypothesized that the observed increase of CT infections in official reports of infectious diseases is a consequence of increased testing of different target groups and does not reflect an increase in disease frequency. Therefore, we requested major diagnostic laboratories to provide denominator data in order to calculate and interpret positivity rates of CT in the canton of Basel-Stadt. Basel-Stadt is one of 26 cantons of Switzerland located in the northwest and considered to be urban. The canton, with 193627 inhabitants in 2010, 32\% of whom are non-Swiss citizens, consists of three civil parishes. About $90 \%$ of the canton's population resides in the city of Basel, the third biggest city in Switzerland [12].

\section{METHODS}

Positivity rate was defined as the number of positive tests divided by the total number of tests performed. With our data, we cannot use prevalence or incidence measures to describe the epidemiology of CT since the denominator is the number of tests performed and not the number of individuals tested. Hence, and in accordance with other studies [10], we use the term 'positivity'.

In 2010, a total of 13 laboratories reported CT cases from residents of the canton of Basel-Stadt to the SFOPH. Of those, seven laboratories reported at least two CT cases from Basel-Stadt and were asked to provide data on gender, year of birth, canton of residence, test date, test result and test method of all subjects tested for CT in the time period 2002-2010. One private sector laboratory declined participation. Four laboratories were from the private sector and provided all requested information. Two were from the public sector and provided complete data for the years 2008-2010. The six laboratories were based in four different cantons of Switzerland (Basel-Stadt, Basel-Land, Zürich, St Gallen). Data from one laboratory had to be entered into an electronic database (Microsoft Access 2002, Microsoft Corporation, USA). All other laboratories provided computerized data.

One third of our data from Basel-Stadt was from a public hospital laboratory even though this laboratory only provided data for 3 years. Given the potentially different client and patient profile in hospital laboratories, including those data in the analysis could obscure a time trend in positivity rates. Therefore, laboratories not providing data for the whole time period were not included in the main analysis.

All collected data were analysed using Stata v. 10.1 (StataCorp., USA). Data analysis and statistical testing was planned a priori. Univariable logistic regression was used to assess whether test year, age group, sex, laboratory and test method were significantly associated with the test result. We included sex and age group and all variables that were significantly associated with the test outcome in the univariable analysis at $P<0.2$ in a multivariable logistic regression. Additionally we included an interaction term for age group and sex in the multivariable model, because females might be infected at different ages than males. We decided not to stratify by sex as we expected sample size to be too small, especially in the male subject group.

The following groups were defined as reference categories: sex 'male'; age ' $\geqslant 40$ '; test year ' 2010 '; laboratory 'Lab D'; and test method 'Cobas Amplicor CT/NG test'.

Inclusion criterion for records was residency of patients in the canton of Basel-Stadt. Records with missing test results were excluded. Records with missing age or sex were excluded in the logistic regression model.

The univariable and multivariable analyses were performed with the original dataset and repeated with a cleaned dataset. In cleaning the data we identified double entries and repeated tests. Records were defined as repeated tests if (i) they were from the same patient entered on the same day from duplicate samples and (ii) if two or more records had identical 


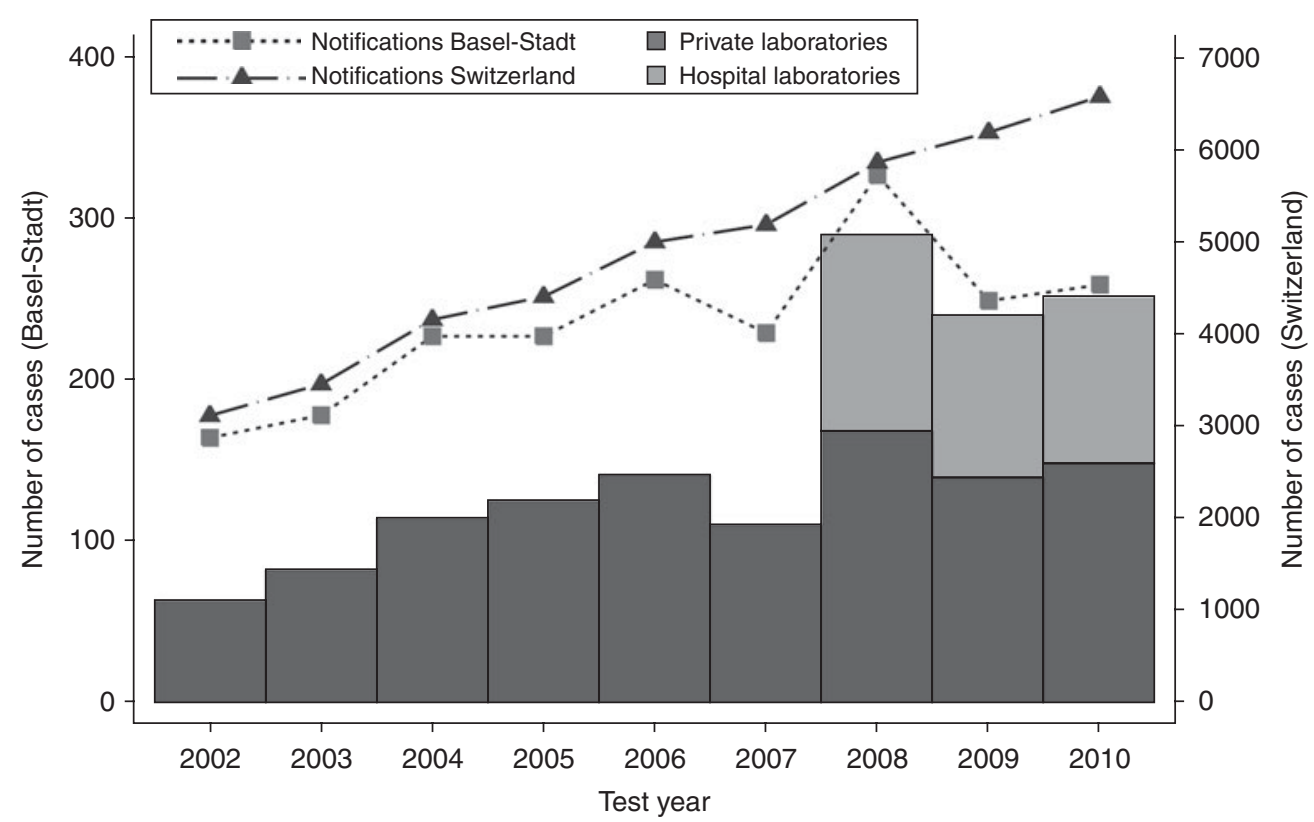

Fig. 1. Absolute number of Chlamydia cases registered at SFOPH for the canton Basel-Stadt and Switzerland (lines) and absolute number of Chlamydia cases (from Basel-Stadt) identified by participating laboratories (bars; left axis) between 2002 and 2010. (Note: total numbers of Chlamydia cases reported by participating laboratories do not account for all cases registered for Basel-Stadt at SFOPH.)

patient identification numbers and a second test on the same patient who tested positive was repeated within 14 days after the initial test with no negative test result in this period. In case of discordant test results the positive result was retained in the analysis.

We calculated CT positivity rates for different age groups and sex. To assess the representativeness of our data we compared the number of CT cases in our data to the cases published by SFOPH.

To assess whether the relative testing frequency between age groups remained constant over time we performed multinomial logistic regressions stratified by sex.

\section{Ethical statement}

This study used data from publically available sources of the SFOPH or fully anonymized data from private and public sector diagnostic laboratories.

\section{RESULTS}

In Switzerland, the number of notified Chlamydia cases increased from 3111 (164 in Basel-Stadt) in 2002 to 6575 (259) in 2010 (Fig. 1).

We obtained 32034 laboratory records on CT testing for the years 2002-2010 in the canton of BaselStadt from six laboratories. Number of tests (and positive tests) reported by the individual laboratories were: 61 (6), 1236 (89), 3232 (187), 4089 (198), 11490 (350) and 11926 (665) over the study period. Our data represent about $45 \%$ of the CT cases in Basel-Stadt published every year by SFOPH (Table 1). Including the tests performed by public laboratories in the years 2008-2010, our data represent between $89 \%$ and $97 \%$ of the SFOPH-registered cantonal CT cases.

\section{Characteristics of laboratories and tested population}

CT testing methods and test performance changed over the study period. Two laboratories did not change the method used for Chlamydia testing during the study period, while two laboratories changed once. However, all but one laboratory used NAATs over the whole period (Table 2). All NAATs applied by the participating laboratories detected DNA of CT.

From 2002 to 2010 the number of tests performed on Basel-Stadt residents increased substantially in the four private laboratories (providing complete data), totalling 1395 in 2002 and 3169 in 2010, resulting in 67 and 155 Chlamydia-positive tests, respectively.

Sixty-three $(0 \cdot 2 \%)$ records were excluded from the overall analysis because of missing test results. 
Table 1. Absolute numbers of reported Chlamydia trachomatis cases in the canton of Basel-Stadt (2002-2010) comparing laboratory-based* and SFOPH† records

\begin{tabular}{|c|c|c|c|c|c|c|c|c|c|}
\hline & 2002 & 2003 & 2004 & 2005 & 2006 & 2007 & 2008 & 2009 & 2010 \\
\hline Cases recorded by $\mathrm{SFOPH} \dagger$ & 164 & 178 & 227 & 227 & 262 & 229 & 327 & 249 & 259 \\
\hline Cases reported by four private laboratories $*+$ & 63 & 82 & 114 & 125 & 141 & 110 & 168 & 139 & 148 \\
\hline$\%$ reported by private laboratories & $38 \%$ & $46 \%$ & $50 \%$ & $55 \%$ & $54 \%$ & $48 \%$ & $51 \%$ & $56 \%$ & $57 \%$ \\
\hline Cases reported by two public sector laboratories & & & & & & & 122 & 101 & 103 \\
\hline$\%$ reported by public sector laboratories & & & & & & & $37 \%$ & $41 \%$ & $40 \%$ \\
\hline Total number of cases reported by laboratories & 63 & 82 & 114 & 125 & 141 & 110 & 290 & 240 & 251 \\
\hline$\%$ total reported & $38 \%$ & $46 \%$ & $50 \%$ & $55 \%$ & $54 \%$ & $48 \%$ & $89 \%$ & $96 \%$ & $97 \%$ \\
\hline
\end{tabular}

* Data of two hospital laboratories were obtained for 3 years only and are, therefore, listed separately.

$\uparrow$ SFOPH (Swiss Federal Office of Public Health), unpublished data.

* Records classified as repeated tests were excluded (the time interval of 90 days between two tests was applied to make numbers comparable to the SFOPH data source).

Table 2. Diagnostic method used to test for Chlamydia trachomatis infections in participating laboratories

\begin{tabular}{|c|c|}
\hline Laboratory & Test method used \\
\hline \multirow[t]{3}{*}{ A } & 2002-2003: LCR* (external laboratory) \\
\hline & 2002-2004: OIA $\dagger$ (BioStar OIA Chlamydia, Inverness Medical, UK) \\
\hline & 2002-2010: PCR $\$$ (external laboratory) \\
\hline $\mathrm{B}$ & kPCR $\S\left(\right.$ Versant ${ }^{\circledR}$ CT/GC DNA 1.0 assay, Siemens, Germany) \\
\hline $\mathrm{C}$ & PCR (Cobas Amplicor CT/NG test, Roche, USA) \\
\hline \multirow[t]{2}{*}{$\mathrm{D}$} & 2002-Jan. 2008: PCR (Cobas Amplicor CT/NG test, Roche, USA) \\
\hline & Feb. 2008-2010: PCR (Abbott Real Time CT/NG assay, USA) \\
\hline $\mathrm{E}$ & 2008-2010: PCR (Cobas Amplicor CT/NG test, Roche, USA) \\
\hline \multirow[t]{2}{*}{$\mathrm{F}$} & 2008-Feb. 2010: PCR (Cobas Amplicor CT/NG test, Roche, USA) \\
\hline & March-Dec. 2010: PCR (Abbott Real Time CT/NG assay, USA) \\
\hline
\end{tabular}

In total, $50(0 \cdot 2 \%)$ records (two positive, 48 negative) were not used for the logistic regression because of missing information on age or sex.

Median age of patients tested was 30 years (range $<1-99$ years) and differed significantly between the laboratories (Kruskal-Wallis test, $P=0 \cdot 0001$ ). Of females, $74 \%$ of CT tests were performed in the 20-39 years group (Fig. 2). For males, the age distribution peaked in slightly older age groups. More than half $(56 \%)$ of tests were performed on males aged $25-44$ years.

While $54 \%$ of positive patients were aged 20-29 years, these represent only $38 \%$ of all tests performed for both sexes within this age group. Positive test results were found most frequently for women aged 15-34 years and men aged 20-39 years.
Females were tested more often than males with proportions tested for CT per year ranging from $80 \%$ to $89 \%$ compared to $11-20 \%$ for men.

\section{Positivity rates}

CT was detected in $821 / 17558$ female samples $(4 \cdot 7 \%)$ and in $317 / 2849$ male samples $(11 \cdot 1 \%)$. Figure 3 shows the time trend in positivity rates for sex and laboratory. In both private and public sector laboratories, positivity rates were stable between 2002 (2008) and 2010. However, the positivity rate in the public hospital laboratories was lower than in the private laboratories.

Univariable logistic regression revealed that sex, age group, test year, laboratory and test method 


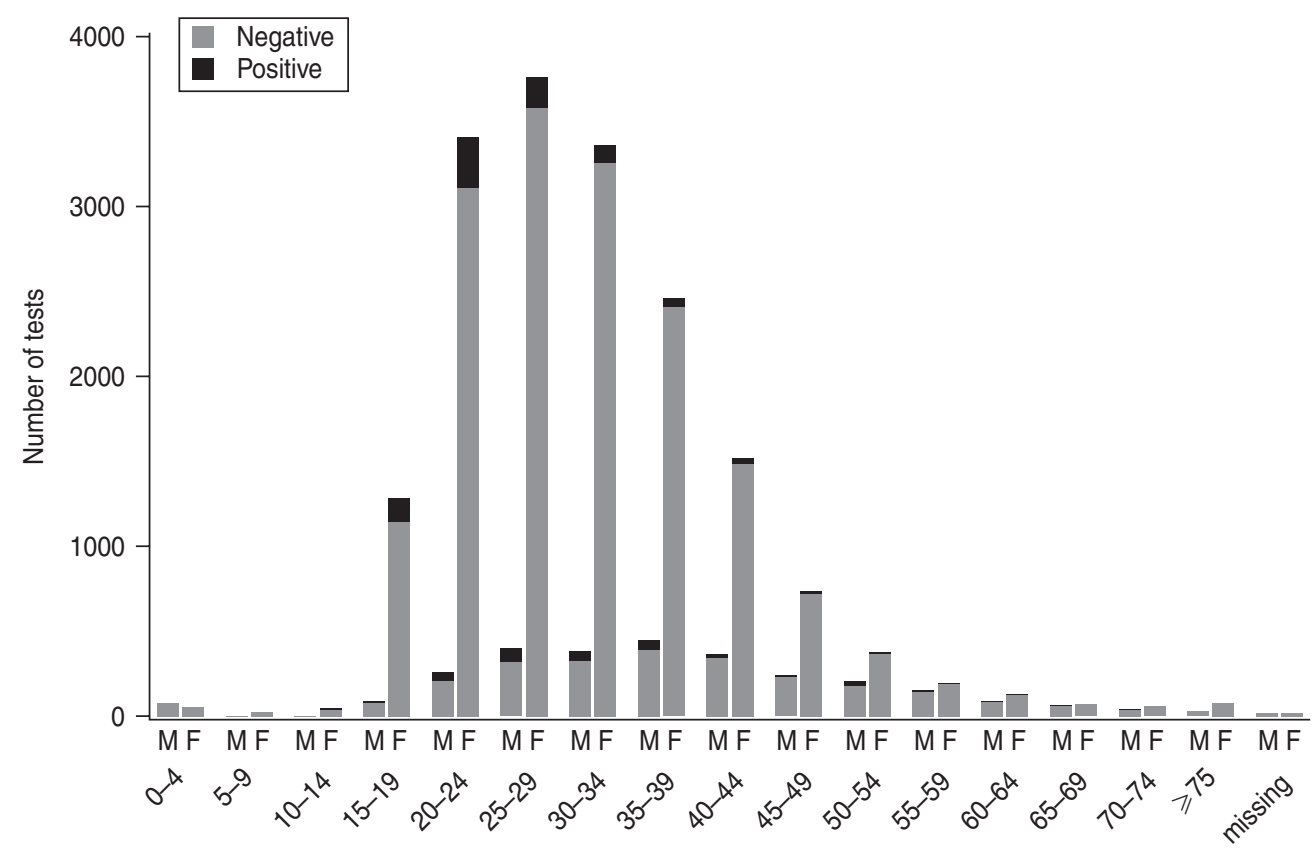

Fig. 2. Test results according to age and sex of people living in Basel-Stadt, tested for Chlamydia trachomatis infection by participating laboratories, 2002-2010. M, Males; F, Females [records with missing test result $(n=63)$ and records with missing information on sex $(n=13)$ are not shown].

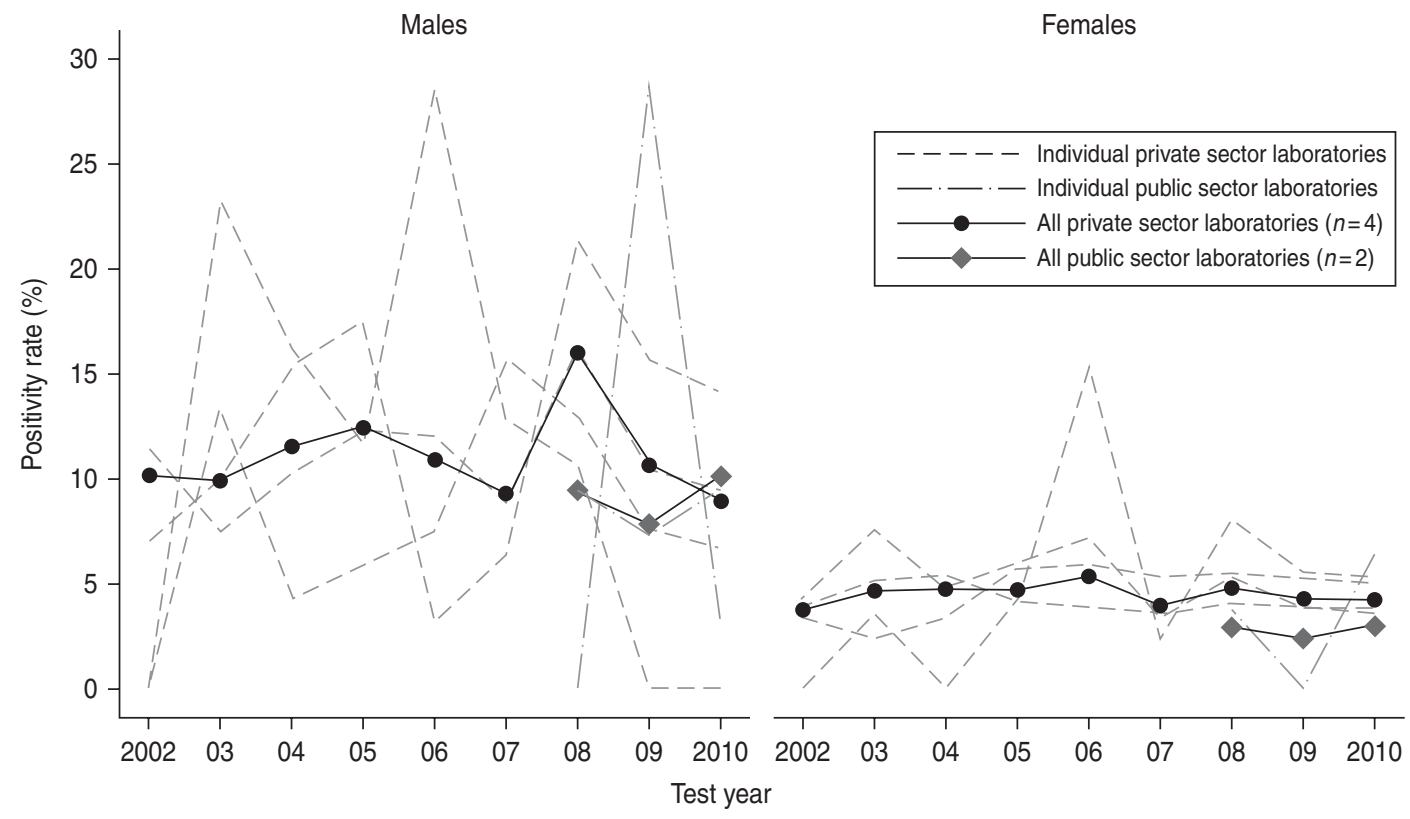

Fig. 3. Time trend in Chlamydia positivity rates in laboratories, by sex. Rates adjusted for age using direct standardization.

were significantly associated with the test result at the $20 \%$ significance level (Table 3). Therefore, all variables except 'test method' were included in the multivariable analysis. The test method was excluded because of partial collinearity with the variable 'laboratory'.
Adjusting for other factors, the year of CT testing remained a significant determinant of a positive test result, although no clear time trend was observed. In the years 2006 and 2008, the probabilities (odds) of testing Chlamydia-positive were significantly higher than in 2010 (annual $P$ values $<0 \cdot 05$ ). 
Table 3. Factors associated with a positive test result for Chlamydia trachomatis in Basel-Stadt residents (crude and adjusted odds ratios)

\begin{tabular}{|c|c|c|c|c|c|}
\hline & $N^{*}$ & OR $(95 \% \mathrm{CI})$ & $P$ value $\dagger$ & aOR $\$(95 \%$ CI $)$ & $P$ value $\dagger$ \\
\hline Sex & 20370 & & $<0.0001$ & & $<0 \cdot 0001$ \\
\hline Male & 2832 & 1 & & 1 & \\
\hline Female & 17538 & $0.39(0.34-0.45)$ & & $0 \cdot 26(0 \cdot 18-0 \cdot 38)$ & \\
\hline Age group, yr & 20370 & & $<0 \cdot 0001$ & & $<0 \cdot 0001$ \\
\hline $0-14$ & 199 & $0 \cdot 19(0 \cdot 03-1 \cdot 38)$ & & - & \\
\hline $15-19$ & 1365 & $4 \cdot 52(3 \cdot 50-5 \cdot 83)$ & & 一 & \\
\hline $20-29$ & 7828 & $3 \cdot 24(2 \cdot 63-3 \cdot 97)$ & & - & \\
\hline $30-39$ & 6648 & $1 \cdot 58(1 \cdot 27-1 \cdot 98)$ & & - & \\
\hline$\geqslant 40$ & 4330 & 1 & & - & \\
\hline Test year & 20370 & & $0 \cdot 018$ & & $0 \cdot 050$ \\
\hline 2002 & 1392 & $0.96(0 \cdot 72-1 \cdot 30)$ & & $0.93(0.69-1 \cdot 26)$ & \\
\hline 2003 & 1526 & $1 \cdot 17(0 \cdot 89-1 \cdot 53)$ & & $1 \cdot 12(0 \cdot 85-1 \cdot 47)$ & \\
\hline 2004 & 1941 & $1 \cdot 27(0 \cdot 99-1 \cdot 62)$ & & $1 \cdot 21(0 \cdot 94-1 \cdot 56)$ & \\
\hline 2005 & 2193 & $1 \cdot 23(0.97-1.56)$ & & $1 \cdot 21(0 \cdot 95-1 \cdot 55)$ & \\
\hline 2006 & 2272 & $1 \cdot 32(1 \cdot 05-1 \cdot 67)$ & & $1 \cdot 30(1 \cdot 03-1 \cdot 65)$ & \\
\hline 2007 & 2420 & $0.97(0 \cdot 76-1 \cdot 24)$ & & $0.96(0 \cdot 75-1 \cdot 24)$ & \\
\hline 2008 & 2639 & $1 \cdot 37(1 \cdot 09-1 \cdot 71)$ & & $1.33(1.06-1 \cdot 67)$ & \\
\hline 2009 & 2828 & $1 \cdot 05(0 \cdot 83-1 \cdot 32)$ & & $1 \cdot 08(0 \cdot 85-1 \cdot 36)$ & \\
\hline 2010 & 3159 & 1 & & 1 & \\
\hline Laboratory & 20370 & & $0 \cdot 009$ & & $0 \cdot 038$ \\
\hline A & 1207 & $1 \cdot 34(1 \cdot 07-1 \cdot 69)$ & & $1 \cdot 37(1 \cdot 08-1 \cdot 74)$ & \\
\hline B & 3230 & $1 \cdot 04(0 \cdot 88-1 \cdot 22)$ & & $1 \cdot 12(0 \cdot 94-1 \cdot 33)$ & \\
\hline $\mathrm{C}$ & 4079 & $0 \cdot 86(0 \cdot 73-1 \cdot 01)$ & & $1 \cdot 15(0.97-1 \cdot 36)$ & \\
\hline $\mathrm{D}$ & 11854 & 1 & & 1 & \\
\hline Test method & 20370 & & $0 \cdot 035$ & & \\
\hline Biostar OIA & 46 & $0 \cdot 39(0 \cdot 05-2 \cdot 85)$ & & 一 & \\
\hline LCR & 53 & $0 \cdot 69(0 \cdot 17-2 \cdot 85)$ & & - & \\
\hline PCR & 1108 & $1 \cdot 48(1 \cdot 17-1 \cdot 88)$ & & 一 & \\
\hline Versant kPCR & 3230 & $1 \cdot 08(0 \cdot 92-1 \cdot 28)$ & & 一 & \\
\hline Abbott PCR & 4347 & $1 \cdot 03(0 \cdot 88-1 \cdot 20)$ & & - & \\
\hline Cobas Amplicor PCR & 11586 & 1 & & - & \\
\hline Males aged, yr & 2832 & & - & & $<0.0001$ \\
\hline $0-14$ & 79 & - & & 一 & \\
\hline $15-19$ & 85 & - & & $1.96(0.94-4.09)$ & \\
\hline $20-29$ & 661 & - & & $4 \cdot 54(3 \cdot 31-6 \cdot 22)$ & \\
\hline $30-39$ & 826 & - & & $2 \cdot 58(1 \cdot 87-3 \cdot 57)$ & \\
\hline$\geqslant 40$ & 1181 & - & & 1 & \\
\hline Females aged, yr & 17538 & & - & & $<0 \cdot 0001$ \\
\hline $0-14$ & 120 & - & & $0 \cdot 51(0 \cdot 07-3 \cdot 76)$ & \\
\hline $15-19$ & 1280 & - & & $7 \cdot 99(5 \cdot 69-11 \cdot 21)$ & \\
\hline $20-29$ & 7167 & - & & $4 \cdot 76(3 \cdot 51-6 \cdot 44)$ & \\
\hline $30-39$ & 5822 & - & & $1 \cdot 87(1 \cdot 35-2 \cdot 60)$ & \\
\hline$\geqslant 40$ & 3149 & - & & 1 & \\
\hline
\end{tabular}

OR, Odds ratio; aOR, adjusted odds ratio; $\mathrm{CI}$, confidence interval.

$* N$, Number of observations (tests).

$\dagger$ From likelihood ratio test.

$\$$ Adjusted odds ratio; adjusted for sex, age group, test year, laboratory and sex $\times$ age group interaction.

The association between age and CT positivity was modified by sex. In males, the adjusted odds ratios (ORs) of testing positive were $2 \cdot 58[95 \%$ confidence interval (CI) $1 \cdot 87-3 \cdot 57], 4 \cdot 54(95 \%$ CI $3 \cdot 31-6 \cdot 22)$ and $1.96(95 \%$ CI $0.94-4.09)$ for the 30-39, 20-29 and 15-19 years age groups, respectively, compared to the 


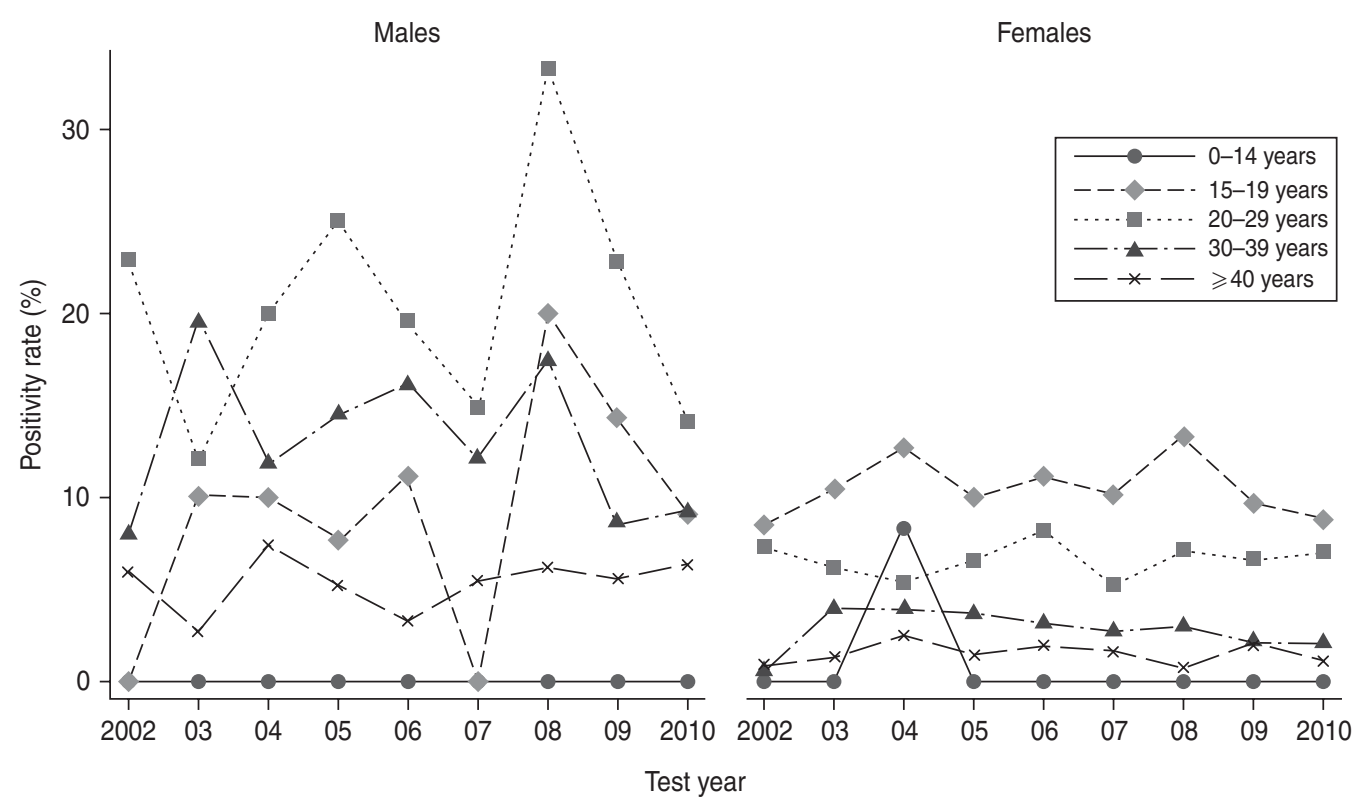

Fig. 4. Time trend in Chlamydia positivity rate in different age groups, by sex.

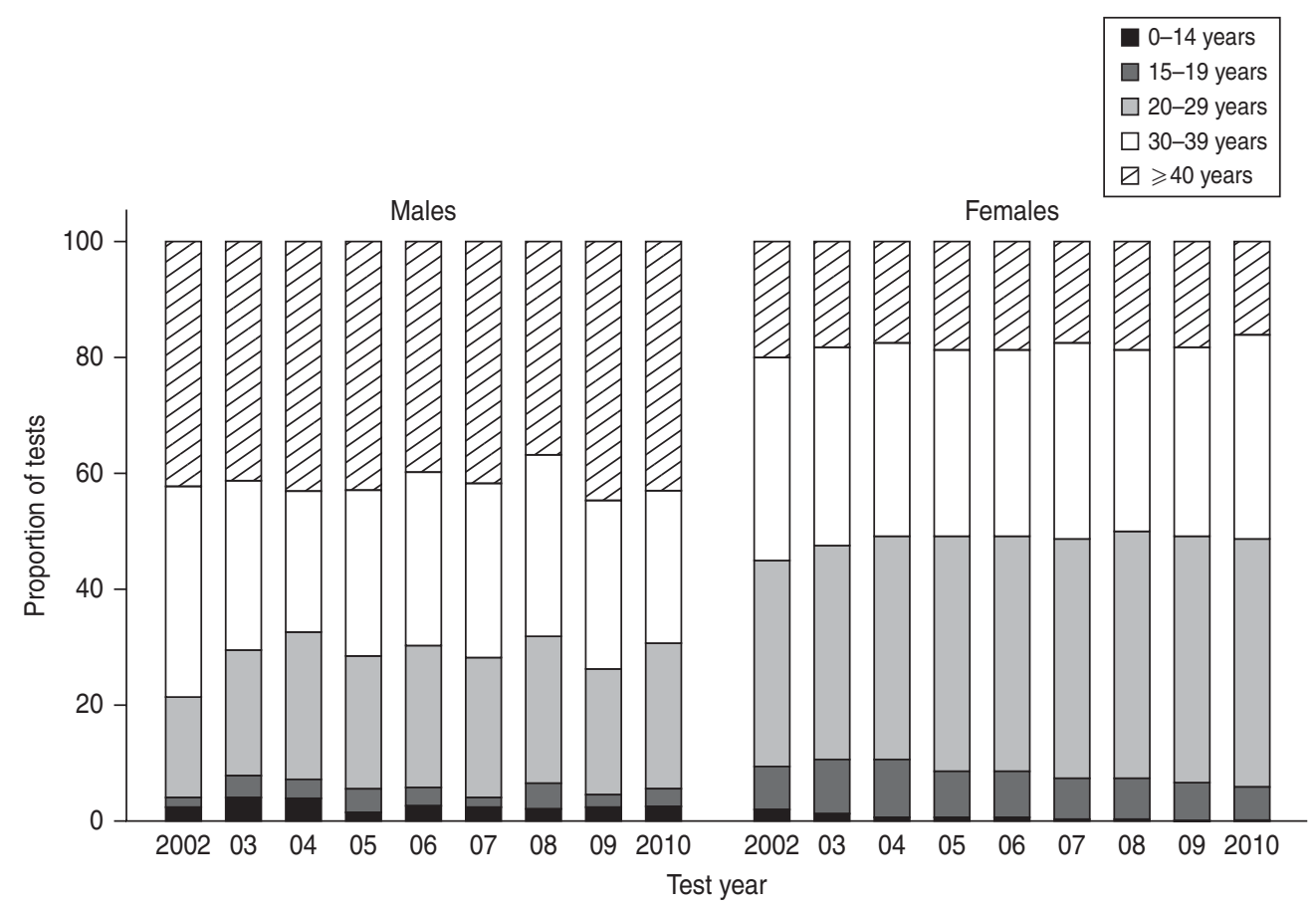

Fig. 5. Proportions of tests performed in different age groups over time, by sex.

reference group ( $\geqslant 40$ years). Compared to females in the $\geqslant 40$ years age group, women aged $30-39,20-29$ and $15-19$ years had adjusted ORs of $1.87(95 \% \mathrm{CI}$ $1 \cdot 35-2 \cdot 60), 4 \cdot 76(95 \%$ CI $3 \cdot 51-6 \cdot 44)$ and $7 \cdot 99(95 \%$ CI $5 \cdot 69-11 \cdot 21)$, respectively. Female sex reduced the probability of a positive test result by $74 \%(95 \% \mathrm{CI}$ 62-82) (Table 3). None out of 79 boys ( $0-14$ years age group) tested for Chlamydia had a positive result and
$1 / 120$ tested girls of the same age group was found positive.

Positivity rates of different age groups stratified by sex over the 9 years indicate that rates in females vary only slightly across the years (Fig. 4). In contrast, in males the positivity rates changed annually.

In Figure 5 we display the proportions of tests performed by age groups and sex from 2002 to 2010. 
Results from the multinomial logistic regression showed that the proportion of tests performed on males in the $15-19$ and $\geqslant 40$ years age groups remained stable over the 9 years (data not shown). Compared to the $\geqslant 40$ years age group, the proportion of tests for males aged $0-14$ and 30-39 years decreased and the proportion of tests for males aged 20-29 years increased, but all of them not significantly. In females, the proportion aged 30-39 years remained stable from 2002 to 2010 while the proportion of the $\geqslant 40$ years age group decreased marginally, the proportions of age groups 0-14 and 15-19 years decreased significantly and that of females aged 20-29 years increased significantly.

The logistic regression analysis was repeated after exclusion of $63(0.3 \%)$ records classified as repeated tests. Results changed very little and interpretation would not have changed at all. Therefore, we do not show those results.

\section{DISCUSSION}

We investigated whether the observed increase in CT case notifications during the past decade was associated with increasing positivity rates. In contrast to other European countries, in Switzerland there were no official screening programmes or national guidelines on CT testing and case management during the study period from 2002 to 2010 .

We found that CT positivity, even though significantly associated with year of testing, did not increase between 2002 and 2010 nor did it exhibit a clear trend in time. This phenomenon can be explained given that the $2 \cdot 5$-fold increase in the absolute number of CT cases observed in our data was accompanied by a 2.5-fold increase in the total number of Chlamydia tests performed. Quality of test methods is assumed to be very similar between laboratories without notably changing during the study period as all but one laboratory used NAAT techniques over the whole study period. Those diagnostic methods have very similar sensitivities and specificities. Nevertheless, differences between laboratories exist and are likely to be linked to different client profiles; in the hospital setting, patients may be more likely to harbour a CT infection (acute care for venereal complaints, lower socioeconomic status in outpatient units, higher proportion of non-Swiss patients) than patients in private practices (predominantly acute care and opportunistic testing during the periodical gynaecological examination of low-risk groups). In contrast, in the hospital settings, tests are prescribed in many instances. Heterogeneity in test-prescribing behaviour also exists among private practitioners [13]. Private laboratories serve predominantly private practices.

The increase in absolute numbers of tests performed over the years can be explained by increased prescription of tests by physicians and to some extent by an increase of overall testing volume within the four laboratories. An increase in test prescriptions may have different underlying reasons: either test inclusion criteria used by the physicians changed over time, leading to more people fulfilling those criteria. If test prescription criteria remained unchanged, the increase in the number of tests performed can be explained by a change in population behaviour with more people meeting the same testing criteria.

\section{Gender imbalance in chlamydial testing}

Overall, $87.5 \%$ of CT tests over the past 9 years were performed on women in the canton of Basel-Stadt. Apart from symptom-based testing and treatment in medical practice in both genders and more frequent testing in risk groups, women are additionally tested in Switzerland during regular gynaecological checkups, even though a Chlamydia test is not included routinely. According to the Swiss Health Survey in $2007,79.8 \%$ of women consulted a gynaecologist in the preceding 2 years and $56.4 \%$ in the preceding 12 months. For men the frequency of interaction with an urologist was unfortunately not assessed by the Swiss national survey [14]. We presume that young men do not routinely consult health services for medical check-ups but rather seek help and contact a specialist based on perceived morbidity, signs and symptoms. A study in attendees of a sexual health clinic in New Zealand found that asymptomatic patients seeking STI testing were significantly more likely to be females than males [15].

A CT infection is more often asymptomatic in females [2,3]. Given different help-seeking patterns in males (symptom-based) and females (screeninglike), CT positivity, therefore, represents two different measures for males and females. For males, positivity measures mostly acute infections (thus, resembling incidence) while for females, positivity may rather represent a prevalence measure; consequently, we are dealing with two different populations. However, neither female nor male positivity rates represent population prevalence or incidence rates since the denominator does not equate the population at risk. 
Data from the NNSID reporting absolute case numbers indicate that $\mathrm{CT}$ infections predominantly occur in women $(70 \%)$. This may further draw the physician's attention to seek for CT infections in females. However, to conclude from this gender imbalance in reported $\mathrm{CT}$ cases to a higher prevalence in females could be misleading because the populations tested differ. Worldwide, there are several studies showing no significant gender differences in prevalence [16-18]. Furthermore, it is common practice to treat sexual partners of infected patients without testing. As females are tested more often, this may lead to more (asymptomatic) male partners being treated without testing and may, thus, account for underrepresentation of male cases. Intensified testing in women could be explained by more frequent testing of risk groups such as pregnant women, women planning contraceptive coil implantations or women undergoing surgical termination of pregnancy, as it is current practice in health-care provision. Additionally, Chlamydia morbidity due to long-term sequelae is higher in females than in males [19]. Whether age at first sexual intercourse plays a role in observed gender differences remains unclear. However, there is only weak evidence that age at first sexual intercourse is a risk factor for chlamydial infection [20].

\section{Positivity rates}

Given the differential testing practice between males and females, it is not surprising that there is a big gender difference in positivity rates. Furthermore, we do not know to what extent physicians treat patients without testing and whether this is done differentially for males and females. According to Paget \& Zimmermann STI diagnoses made by Sentinella physicians were based on laboratory tests in only $40 \%$ of cases [21]. With the new national prevention campaign for HIV and other STIs, the SFOPH published case management guidelines for primary-care physicians [22]. These recommendations consider treatment of infections without laboratory confirmation if a symptomatic diagnosis is confirmative or if the partner is treated for the same infection. Moreover, financial issues could play a role in treating patients without laboratory confirmation, especially in young, non-pregnant adults. The yearly CT positivity rate varied without a clear time trend between $4.7 \%$ (2007) and 6.6\% (2008). Bender et al. found positivity rates in Denmark, Sweden and New Zealand to be slightly higher and increasing over time from about $5 \%$ in 1999 to $8 \%$ in 2008 [10]. Like Switzerland, these countries had no on-going screening programmes but Chlamydia case management guidelines existed, recommending Chlamydia testing at least in one group of asymptomatic patients. Comparable guidelines have only existed in Switzerland since 2011. Even though screening programmes or national guidelines can impact on positivity rates, monitoring positivity rates is not sufficient to evaluate the effectiveness of such programmes or guidelines.

Positivity rates in young adults (aged $<25$ years) tested during the fifth year of the English National Screening Programme (2007/2008) were $7 \cdot 6 \%$ in men and $9.3 \%$ in women. By contrast, in our sample positivity rates in those aged 15-24 years were $18 \cdot 1 \%$ in men and $9 \cdot 3 \%$ in women over the entire study period. This substantial difference in men between the two countries is probably explained by the difference in testing practices. Such differences are not observed in women suggesting that the current practice of CT testing in Switzerland may resemble a screening-like approach in the absence of an official screening programme. There is no evidence to assume a higher CT prevalence in Switzerland than in the UK: population-based prevalence was estimated at $0-4.9 \%$ in the UK [23]. The prevalence in Swiss men at army recruitment was $1 \cdot 2 \%$, the most accurate prevalence estimate available for young men aged between 18 and 26 years in Switzerland [8]. A study by Swiss sentinel gynaecologists found a prevalence of $1.3 \%$ in pregnant women and $2.8 \%$ in women who went for a gynaecological check-up [24].

From a 1-year laboratory-based Swiss study in 2009 we calculated a CT positivity rate of $4.0 \%$ in females and $6.4 \%$ in males [25]. These figures are comparable to our observed positivity rates of $4.5 \%$ in females and $10 \cdot 2 \%$ in males in 2009 .

\section{Testing practices}

A stable positivity rate does not necessarily mean that incidence is stable and, vice versa, an increasing positivity rate could occur without a true increase in incidence. All those measures highly depend on testing practices of physicians and on the population tested.

We calculated positivity rates because we have different test groups (males representing rather incidence and females as a proxy for prevalence) and cannot conclude on the true epidemiological situation. 
In order to equate the trend in positivity rates with trends in prevalence or incidence rates we would need to assume that the testing practice in the two groups as well as sexual risk behaviour remained constant over time. We only have data on age and sex to investigate this assumption. Figure 5 describes the relative testing frequencies in each age group. For males, relative testing frequency did not change across age groups. However, in females, there was a tendency over the observed 9-year period to perform more tests in one age group (20-29 years) and less in others $(0-14,15-19, \geqslant 40$ years). It is, therefore, possible that the target population for Chlamydia tests changed, especially in females, which in turn leads to different pre-test probabilities of being infected. This could mean that the trend observed in the positivity rate could diverge from the trend of the real CT incidence (males) or CT prevalence (females). The latter two can only be obtained from random populationbased surveys.

Median age of negatively tested persons was 31 compared to a median age of 26 years in positively tested persons. An explanation for this difference in median age is that pregnant women are tested frequently and only up to $38 \%$ of live births in Switzerland occur in mothers aged 20-29 years, while as many as $60 \%$ of live births occur in those aged $30-39$ years [12].

\section{Treatment and repeated testing}

We could identify only $63(0 \cdot 3 \%)$ records as repeated tests considering a second $\mathrm{CT}$ test performed within 14 days as a repeated test. Excluding these from the analysis did not change the interpretation of the results. Similarly, applying the SFOPH criteria of a time interval of 90 days differentiating a repeated $v s$. a new infection did not change the results (data not shown).

Repeated infections are not likely to be due to treatment failure. The most commonly used antibiotics for CT therapy (azithromycin, doxycycline) have been shown to be equally efficacious with cure rates of $97-98 \%$ [26]. Therefore, persistent infections are more likely due to compliance issues or immediate re-infection because of on-going sexual contacts with infectious partners. Further, persistence of DNA from dead chlamydial bacteria may lead to falsepositive results for up to 3 weeks following treatment [27-29].

Our study has some limitations. We enrolled six of seven major laboratories into the study. However, two laboratories could not provide data for the whole time period and were, therefore, excluded from the main analysis. Even though our data represent only half of the cantonal Chlamydia infections reported to SFOPH, our data are likely to reflect the situation in Basel-Stadt. This is consistent with the findings from the regression model using data from all six laboratories for the years 2008-2010: the positivity rate did not change in the past few years in Basel-Stadt.

Interpretation of the total number of $\mathrm{CT}$ tests performed is hampered because the increase of CT testing could be a consequence of structural growth of individual diagnostic laboratories, thus, of increased overall testing volume rather than of an increased CT testing frequency. This is supported by the fact that participating laboratories reported higher percentages of CT cases to SFOPH over time (Table 1).

Repeated tests could not always be identified as the Swiss health information system does not (yet) utilize unique patient identification numbers. The use of test methods of different sensitivities and specificities in laboratories could explain some of the variation between diagnostic laboratories; however, choice of CT test material, i.e. urine, urethral or vaginal swab may have a larger impact on the sensitivity and specificity of test outcomes than the choice of a NAAT method [25]. The recent use of a combined CT and Neisseria gonorrhoea test could also potentially result in a rising detection rate of CT infections and, therefore, in increasing numbers of cases as observed in the NNSID. Additional CT diagnoses resulting from a simultaneous testing for CT and $N$. gonorrhoea, as done in one private and one hospital laboratory, could not be identified. Finally, test-prescribing behaviour of physicians relating to the control of treatment outcome, screening of pregnant women or other 'risk' groups, or as an effect of national control endeavours may have changed over time. The 2009 Swiss HIV/ AIDS prevention campaign called 'LOVE LIFE STOP AIDS' (www.lovelife.ch) did not have a measurable impact on Chlamydia positivity rates in 2009/2010. Messages on STIs in general and chlamydial infections in particular are only explicitly featured in the subsequent recent campaign in 2011 ('LOVE LIFE'; www.lovelife.ch).

\section{CONCLUSION}

Data from the NNSID provide no denominator data on CT and other infectious diseases limiting epidemiological interpretation of publically available 
health information. Considering denominator data from diagnostic laboratories for residents of the canton of Basel-Stadt, the observed increase of CT cases in the NNSID may not reflect a higher frequency of this notifiable sexually transmitted infection. We found no increase in Chlamydia positivity in Basel-Stadt over the past 9 years.

Studies from European and other countries, without screening for CT (similar to Switzerland), suggest a true increase of CT positivity rates. This contrasts with the findings of our study and underscores the necessity of obtaining comparable CT data for other Swiss cantons to conclude on recent epidemiological patterns of CT infections for the whole of Switzerland.

This study has shown that great caution is needed when interpreting changes in numbers of disease notifications without knowing the denominator.

\section{ACKNOWLEDGEMENTS}

The authors acknowledge Dr Susanne Graf (Kantonsspital Liestal), Professor Dr Markus Gasser (Medibact), Dr Martin Wyss (team w AG), Dr Marinko Dobec, Dr Pascal Cassinotti, Dr Franz Käppeli (Medica, Medizinische Laboratorien Dr F. Käppeli) and Mr Michael Feuz (University Hospital of Basel) for providing necessary data.

The contributions of Drs Dobec, Cassinotti and Käppeli in writing an earlier draft of the manuscript are appreciated. We thank $\mathrm{Dr}$ Sibil Tschudin (University Hospital of Basel) for sharing her clinical experience and Dr Francisca Morán Cadenas (SFOPH) for providing details on the national surveillance data and advice in the interpretation of our results. Dr Valérie d'Acremont's critical comments and Dr Jan Hattendorf's support in statistical matters are gratefully acknowledged. This research received no specific grant from any funding agency, commercial or not-for-profit sectors.

The participating private and public laboratories provided data only. Authors from those institutions contributed in kind only in interpreting the results and in writing the manuscript. The findings and conclusions of this report are those of the authors and do not necessarily represent the views of the individual institutions.

\section{DECLARATION OF INTEREST}

None.

\section{REFERENCES}

1. Fenton KA, Lowndes CM, ESSTI Network. Recent trends in the epidemiology of sexually transmitted infections in the European Union. Sexually Transmitted Infections 2004; 80: 255-263.

2. Manavi K. A review on infection with Chlamydia trachomatis. Best Practice \& Research. Clinical Obstetrics \& Gynaecology 2006; 20: 941-951.

3. Bébéar C, de Barbeyrac B. Genital Chlamydia trachomatis infections. Clinical Microbiology and Infection 2009; 15: 4-10.

4. van de Laar MJ, Morré SA. Chlamydia: a major challenge for public health [Editorial]. Eurosurveillance 2007; 12: pii $=735$.

5. World Health Organisation. Global prevalence and incidence of selected curable sexually transmitted infections. Geneva: WHO, WHO/HIV AIDS/2001,02.

6. Steiner AS, et al. Chlamydia trachomatis infection in a Swiss prison: a cross sectional study. Swiss Medical Weekly 2010; 140: w13126.

7. Jackson Y, et al. Prevalence and associated factors for Chlamydia trachomatis infection among undocumented immigrants in a primary care facility in Geneva, Switzerland: a cross-sectional study. Journal of Immigrant and Minority Health 2010; 12: 909-914.

8. Baud D, et al. Low prevalence of Chlamydia trachomatis infection in asymptomatic young Swiss men. BMC Infectious Diseases 2008; 8: 45.

9. SR818-101 EpG. Bundesgesetz vom 18. Dezember 1970 über die Bekämpfung übertragbarer Krankheiten des Menschen (Epidemiengesetz), 1974.

10. Bender $\mathbf{N}$, et al. Chlamydia infection, pelvic inflammatory disease, ectopic pregnancy and infertility: crossnational study. Sexually Transmitted Infections 2011; 87: 601-608.

11. Bundesamt für Gesundheit. Sexuell übertragbare Infektionen (STI) in der Schweiz 2006 bis 2008 (http:// www.bag.admin.ch/themen/medizin/00682/00684/01063/ index.html?lang=de). Accessed 28 June 2011.

12. Bundesamt für Statistik. Website Statistik Schweiz (http://www.bfs.admin.ch/bfs/portal/de/index/infothek/ lexikon/lex/0.html) Accessed 5 January 2012.

13. Pletscher F. Determinants of Chlamydia trachomatis infections in the canton Basel-Stadt (M.Sc. thesis). Basel, Switzerland: University of Basel and Swiss TPH, 2011, 100 pp.

14. Bundesamt für Statistik. Gesundheit und Gesundheitsverhalten in der Schweiz 2007. Schweizerische Gesundheitsbefragung. Neuchâtel 2010.

15. Morgan J, Haar J. Who goes to a sexual health clinic? Gender differences in service utilisation. New Zealand Medical Journal 2008; 121: 44-49.

16. European Centre for Disease Prevention and Control. ECDC Guidance. Chlamydia control in Europe. Stockholm: ECDC, 2009.

17. Beydoun HA, et al. Gender and age disparities in the prevalence of chlamydia infection among sexually active adults in the United States. Journal of Women's Health 2010; 19: 2183-2190. 
18. Goulet V, et al. Prevalence of Chlamydia trachomatis: results from the first national population-based survey in France. Sexually Transmitted Infections 2010; 86: 263-270.

19. Carey AJ, Beagley KW. Chlamydia trachomatis, a hidden epidemic: effects on female reproduction and options for treatment. American Journal of Reproductive Immunology 2010; 63: 576-586.

20. Navarro C, et al. Risk factors for genital chlamydial infection. Canadian Journal of Infectious Diseases 2002; 13: 195-207.

21. Paget WJ, Zimmermann HP. Surveillance of sexually transmitted diseases in Switzerland, 19731994: evidence of declining trends in gonorrhoea and syphilis. Sozial- und Präventivmedizin 1997; 42: 3036.

22. Bundesamt für Gesundheit. Empfehlungen zur frühzeitigen Behandlung von sexuell übertragbaren Infektionen (STI) durch erstbehandelnde Ärztinnen und Ärzte: Klinisches Vorgehen bei Beschwerden im Genitalbereich. 2011.

23. Adams EJ, et al. Chlamydia trachomatis in the United Kingdom: a systematic review and analysis of prevalence studies. Sexually Transmitted Infections 2004; 80: 354-362.
24. Paget WJ, et al. National laboratory reports of Chlamydia trachomatis seriously underestimate the frequency of genital chlamydial infections among women in Switzerland. Sexually Transmitted Diseases 2002; 29: 715-720.

25. Sakem B, et al. Diagnostic relevance of simultaneous testing for Chlamydia trachomatis and Neisseria gonorrhoeae. Infection 2011; 39: 231-237.

26. Lau CY, Qureshi AK. Azithromycin versus doxycycline for genital chlamydial infections: a meta-analysis of randomized clinical trials. Sexually Transmitted Diseases 2002; 29 : 497-502.

27. Gaydos CA, et al. Molecular amplification assays to detect chlamydial infections in urine specimens from high school female students and to monitor the persistence of chlamydial DNA after therapy. Journal of Infectious Diseases 1998; 177: 417-424.

28. Morré SA, et al. Monitoring of Chlamydia trachomatis infections after antibiotic treatment using RNA detection by nucleic acid sequence based amplification. Molecular Pathology 1998; 51: 149-154.

29. Roosendaal R, et al. Comparison of different primer sets for detection of Chlamydia trachomatis by the polymerase chain reaction. Journal of Medical Microbiology $1993 ; 38$ : 426-433. 